\title{
SAVING IRRIGATION WATER AND IMPROVING WATER PRODUCTIVITY IN RICE CULTIVATION BY INDUCING GOOD LAND LEVELING AND NEW PLANTING METHOD IN NORTH NILE DELTA, EGYPT

\author{
Metwally, M. A ${ }^{1}$. and Gh. Sh. El-Atawy ${ }^{2}$
}

\section{ABSTRACT}

Two field experiments were conducted at Sakha Agricultural Research Station, Kafr El-Sheikh Governorate, Egypt. during the two successive seasons of 2012 and 2013. The rice cultivar was Sakha 104. The experiment was designed as a split split-plot design with four replicates. Results showed that both submerged depth of 9 and $7 \mathrm{~cm}$ significantly increased grain yield, plant height, number of panicles/hill, number of tillers/hill, panicle length, panicle weight, 1000 grains weight and seasonal applied irrigation water compared to submerged depth of $5 \mathrm{~cm}$ $\left(d_{3}\right)$, there were no significant differences between submerged depth of $d_{1}$ and $d_{2}$. Planting in bottom of beds $\left(M_{3}\right)$ significantly increased grain yield by $8.48 \%$, plant height by $4.38 \%$, number of panicles/hill by $8.34 \%$, number of tillers/hill by $10.58 \%$, panicle length by $16.9 \%$, Panicle weight by $6.62 \%$ and 1000 grains weight by $6.83 \%$, compared to $M_{1}$. The highest mean values of grain yield (12.51 ton $\left.\mathrm{ha}^{-1}\right)$, plant height $(113 \mathrm{~cm})$, No. of panicles/hill (31), No. of tillers/hill (32), panicle length (28cm), panicle weight $(2.98 \mathrm{gm})$ and 1000 grain weight $(30.91 \mathrm{gm})$ were obtained from interaction between $\left(\begin{array}{lllll}L_{2} & x & M_{3} & x & d_{2}\end{array}\right)$. Average amounts of the applied irrigation water were 13885,11519 and $8919 \mathrm{~m}^{3} / \mathrm{ha}^{-1}$, for $\left(M_{1}\right),\left(M_{2}\right)$ and $\left(M_{3}\right)$, respectively. According the highest mean values of productivity of irrigation water $(P I W)\left(1.480 \mathrm{~kg}\right.$ grain $\left.\mathrm{m}^{-3}\right)$ was obtained from combination between $\left(\begin{array}{lllll}L_{2} & x & M_{3} & x & d_{3}\end{array}\right)$, So, method of $\left(M_{3}\right)$ saved about $35.8 \%$ of the irrigation water applied and increased productivity of irrigation water (PIW) by $13 \%$ compared to $\left(M_{1}\right)$.Therefore, method of planting in bottom of beds could be recommended for planting rice crop under laser leveling in North Delta Egypt.

Abbreviations: Productivity of irrigation water (PIW), land leveling (L), Planting method (M) and irrigation water depth (d).

Keywords: rice; irrigation; water saving; water productivity

\footnotetext{
${ }^{1}$ Agric. Eng. Res. Inst., Agric. Res. Center, Giza, Egypt

${ }^{2}$ Soils, Water and Environment Res. Inst., Agric. Res. Center, Giza, Egypt
} 


\section{INTRODUCTION}

$\mathrm{E}$ gypt presently has the highest average rice yield in the world; however, the country's rice yield must be increased by $20 \%$ over the next decade just to maintain current levels of consumption (El-Atawy, 2012).

This will be difficult because the yield level is already high, and because of increasing competition for water with growing water shortages that affect all sectors. Water availability is becoming progressively more limited, as an increasing population makes competing demands for this precious resource. The challenge for agricultural researchers is to find ways to reduce the water consumption in rice production while continue to increase yields.

Improving water productivity (WP) is an important strategy for addressing future water scarcity which is driven particularly by population growth and potential changes in climate and land use. Improving WP in agriculture will reduce competition for scarce water resources, mitigate environmental degradation and enhance food security simply because by producing more food with less water rewards the saved water to other natural and human uses (Rijsberman, 2001 and Molden $\boldsymbol{e t}$ al., 2001).

Furrow-irrigated rice-production systems have recently begun to receive increased attention among rice producers and media outlets. Furrowirrigation can generally saturate the soil and may be similar to floodirrigation (Vories et al., 2002). Vories et al. (2002) observed a 15.6\% yield reduction in furrow-irrigated rice compared to flood-irrigated rice.

Atta (2005) found that by applying the innovative planting method for cv. Sakha 104 obtained the highest grain yield per hectare, compared with traditional planting (3.4\% increment). He also indicated that reduction of the total water applied from $14870 \mathrm{~m}^{3} \mathrm{ha}^{-1}$ to $9545 \mathrm{~m}^{3} \mathrm{ha}^{-1}$, resulted in water saving of $35.8 \%$ of the total water applied and increased water use efficiency from 0.66 to $1.06 \mathrm{~kg} \mathrm{~m}^{-3}$ (60.6\% increment).

Atta et al. (2006) showed that planting in strips of furrows $80 \mathrm{~cm}$ wide resulted in the highest value of grain yield $\left(9.05\right.$ ton $\left.^{-1} \mathrm{~h}^{-1}\right)$, followed by planting in strips of furrows $60 \mathrm{~cm}$ wide $\left(9.00\right.$ ton $^{-1}$ ha $)$ and traditional planting $\left(8.71\right.$ ton $\left.\mathrm{ha}^{-1}\right)$. They also indicated that irrigation water applied 
was 9028.6, 10047.6, and $15628.6 \mathrm{~m}^{3} \mathrm{ha}^{-1}$, and water use efficiency values were $1.0,0.896$ and $0.558 \mathrm{~kg}$ grain $\mathrm{m}^{-3}$ of water applied for planting in stripes of furrows $80 \mathrm{~cm}$ wide, planting in strips of furrows 60 $\mathrm{cm}$ wide and traditional planting, respectively. In comparison with traditional planting, saving water values were $42.23 \%$, and $35.71 \%$ for planting in strips of furrows $80 \mathrm{~cm}$, planting in strips of furrows $60 \mathrm{~cm}$ wide, respectively.

Beecher et al. (2006) showed that rice crop water use was significantly different between the layout-irrigation treatments. The Flat, Bed 5 and Bed 15 treatments had similar input (irrigation + rainfall-surface drainage) water use (mean of $18.3 \mathrm{ML} / \mathrm{ha}$ ). The water use for the Furrow treatment was 17.2 ML/ha and for the Furr/Drip treatment, 15.1 ML/ha. Input WP of the Flat treatment $(0.68 \mathrm{t} / \mathrm{ML})$ was higher than the raised bed treatments, which were all similar (mean $0.55 \mathrm{t} / \mathrm{ML}$ ). This single season experiment showed that high yielding rice crops can be successfully grown on raised beds, but when beds are ponded after panicle initiation, there is no water saving compared with rice grown on a conventional flat layout.

Choudhury et-al. (2007) showed that rice yields on raised beds that were kept around field capacity, 32-42\% lower than under flooded transplanted conditions and $21 \%$ lower than under flooded wet-seeded conditions. Water inputs were reduced by $32-42 \%$ compared with flooded rice, but could also be accomplished with dry seeding on flat land with the same water management. Reduced water inputs and yield reductions balanced each other, so that water productivity was comparable among most treatments

Jagroop Kaur et al. (2007) studied the effects of different planting techniques on the growth, productivity and water saving in paddy. Treatments comprised: transplanting in flat puddle field with 15- or 30day-old seedlings ( 33 plants $\mathrm{m}^{-2}$ ), transplanting in furrows with 15-dayold seedlings ( 22 or 33 plants $\mathrm{m}^{-2}$ ), transplanting in furrows with 30-dayold seedlings ( 22 or 33 plants $\mathrm{m}^{-2}$ ), transplanting on beds with 15-day-old seedlings, transplanting on beds with 30-day-old seedlings (22 or 33 plants $\mathrm{m}^{-2}$ ), direct sowing in rows in flat unpuddled field and direct 
broadcasting. The rice transplanted with 15 - or 30-day-old seedlings and by using 22 or 33 plants $\mathrm{m}^{-2}$ produced statistically similar grain yield. The furrow and bed transplanting saved $119.5 \mathrm{~cm}(39.0 \%)$ irrigation water from puddling to harvest and 44.2 to $50.0 \%$ more water expense efficiency than the recommended practice of flat transplanting under same age (30 days) of seedlings.

El-Atawy (2012) obtained that irrigation water applied in rice fields could be significantly reduced without sacrificing rice yield or without increasing the production cost by using the treatment irrigation water depth $7 \mathrm{~cm}\left(\mathrm{~d}_{2}\right) \times$ transplanting in beds $\left(\mathrm{M}_{2}\right)$. Method of transplanting at bottom of beds $\left(\mathrm{M}_{2}\right)$ increased productivity of irrigation water (PIW) by $45 \%$ than traditional method $\left(\mathrm{M}_{1}\right)$. Therefore, transplanting rice in beds only and keeping it under continuous irrigation $\left(\mathrm{d}_{1} \times \mathrm{M}_{2}\right)$ could be applied by the farmers because it increased (PIW) by 53\% and saved water by $33 \%$ compared to $\mathrm{d}_{1} \times \mathrm{M}_{1}$ in North Delta, Egypt.

The objective of this investigation was to produce more rice with less water under matching planting methods with precision land leveling in North Delta, Egypt.

\section{MATERIALS AND METHODS}

A field experiment was carried out during the two successive rice growing seasons of 2012 and 2013 at Crops Water Requirement Research Field, Sakha Agricultural Research Station, Kafr El-Sheikh Governorate. The site is allocated at 31-07' N Latitude, 30-57'E Longitude with an elevation of about 6 meters above mean sea level. The site represents the conditions and circumstances of North Nile Delta region. The soil of the experimental site was clayey texture and contained $47.1 \%$ clay, $25.6 \%$ silt and $27.3 \%$ sand. The average of the electrical conductivity of soil salinity over $0-60 \mathrm{~cm}$ depth was $1.63 \mathrm{dSm}^{-1}$, the electrical conductivity of irrigation water was $0.54 \mathrm{dSm}^{-1}$. The preceding crop was clover in both seasons.

The experiment was designed as a split split-plot design with four replicates. The main plots were randomly occupied by two land leveling, normal land leveling $\left(\mathrm{L}_{1}\right)$ and laser land leveling with $0.1 \%$ slop $\left(\mathrm{L}_{2}\right)$. While three planting methods treatments were in the sub plots, traditional transplanting in flooded soil $\left(\mathrm{M}_{1}\right)$, transplanting in furrow $\left(\mathrm{M}_{2}\right)$ and 
transplanting in beds $\left(\mathrm{M}_{3}\right)$, and three irrigation depths 9,7 and $5 \mathrm{~cm}$ for $\mathrm{d}_{1}$, $\mathrm{d}_{2}$ and $\mathrm{d}_{3}$, respectively were assigned to sub-sub plots.

The raised rows were $60 \mathrm{~cm}$ distance from mid row to mid another while, the raised beds were $20 \mathrm{~cm}$ high x $45 \mathrm{~cm}$ wide with $80-\mathrm{cm}$ distance from mid bed to mid another. The plots were isolated by ditches of $2.5 \mathrm{~m}$ in width to avoid lateral movement of water.

Rice cultivar was Sakha 104, on may $15^{\text {th }}$ and $20^{\text {th }}$ in 2012 and 2013, respectively, twenty five days old seedlings were transplanted in hills spaced 20 by $20 \mathrm{~cm}$ to give 25 hills $\mathrm{m}^{-2}$ for traditional planting, while transplanted in hills spaced $13.3 \mathrm{~cm}$ apart on two sides of furrow in bottom to give 25 hills $\mathrm{m}^{-2}$ and spaced 10 by $10 \mathrm{~cm}$ in the two rows in bottom of bed to keep population on 25 hills $\mathrm{m}^{-2}$ for beds. Cultural practices were similar to those used in the area.

Rice plants were harvested at 120 days from sowing Data collected were plant height in $\mathrm{cm}$, number of tillers per hill, number of panicles per hill, panicle length in $\mathrm{cm}$, panicle weight in g, 1000-grain weight in $\mathrm{g}$, and rice grain yield ton $\mathrm{ha}^{-1}$ at maturity. The grains were separated from the straw, and the grains were weighed. Grain yield was calculated based on the adjustment to grain moisture content of $140 \mathrm{~g} \mathrm{~kg}^{-1}$ The mean values of some soil Physical, chemical properties and some water constants of the experimental site before cultivation were presented in Table (1).

Table (1): The mean values of some soil Physical, chemical properties and some water constants of the experimental site before cultivation

\begin{tabular}{|c|c|c|c|c|c|c|c|c|c|c|c|c|c|c|c|c|c|}
\hline \multicolumn{3}{|c|}{$\begin{array}{l}\text { Particle size } \\
\text { distribution\% }\end{array}$} & \multirow{2}{*}{$\begin{array}{c}\text { Texture } \\
\text { class }\end{array}$} & \multirow{2}{*}{\begin{tabular}{|c|} 
F.C \\
$\%$
\end{tabular}} & \multirow{2}{*}{$\begin{array}{l}0 \\
0 \\
0 \\
0\end{array}$} & \multirow{2}{*}{$\begin{array}{l}\text { Available } \\
\text { Water\% }\end{array}$} & \multirow{2}{*}{$\begin{array}{l}\text { Bulk } \\
\text { density, } \\
\mathrm{Mg} / \mathrm{m}^{3}\end{array}$} & \multirow{2}{*}{$\begin{array}{c}\mathrm{EC} \\
\mathrm{dSm}^{-1}\end{array}$} & \multirow{2}{*}{$\mathrm{pH}$} & \multicolumn{8}{|c|}{ Soluble ions MeqL $^{-1}$} \\
\hline and & Silt & Clay & & & & & & & & $\mathrm{Ca}^{2+}$ & $\mathrm{Mg}^{2+}$ & $\mathrm{Na}^{+}$ & $\mathbf{K}^{+}$ & $\mathrm{CO}_{3}{ }^{2-}$ & $\mathrm{HCO}_{3}^{-}$ & $\mathrm{Cl}^{-}$ & $\mathrm{SO}_{4}{ }^{2-}$ \\
\hline 27.3 & 25.6 & 47.1 & Clay & 47.0 & 25.3 & 21.7 & 1.19 & 1.63 & 8.15 & 0.30 & 0.10 & 0.76 & 0.02 & - & 0.55 & 0.21 & 0.42 \\
\hline
\end{tabular}

\section{Irrigation water applied (IWA)}

The irrigation water was applied to the experimental plots until reaching the end of the plot length. This was measured and delivered by a constant rectangular weir with steel gates for each plot. The rate of discharge was $0.01654 \mathrm{~m}^{3} / \mathrm{sec}$ at effective head of $10 \mathrm{~cm}$. The amount of applied water for each plot of the studied treatments was calculated by the equation; 


$$
\mathbf{Q}=\mathbf{q} \times \mathbf{t}
$$

Where:

$\mathrm{Q}$ is the volume of water delivered to the plot $\left(\mathrm{m}^{3}\right)$,

$\mathrm{q}$ is the discharge of the weir $\left(\mathrm{m}^{3} / \mathrm{min}\right)$ and

$\mathrm{t}$ is the time of irrigation (min).

\section{Productivity of irrigation water (PIW)}

Productivity of irrigation water (PIW) was calculated according to (Ali $\boldsymbol{e t}$ al., 2007)

$$
\text { PIW= GY/I }
$$

Where PIW in $\left(\mathrm{kg} \mathrm{m}^{-3}\right), \mathrm{GY}$ is grain yield $\left(\mathrm{kg} \mathrm{ha}^{-1}\right)$ and $\mathrm{I}$ is the amount of applied water in $\mathrm{m}^{-3} \mathrm{ha}^{-1}$.

The obtained data were statistically analyzed by analysis of variance. The data of the two seasons showed nearly the same trend, Thus, combined analysis was done according to Gomez and Gomez (1984) .Means of the treatments were compared by the least significant difference (LSD) at 5\% level of significance which developed by Waller and Duncan (1969).

\section{Grain yield and its attributes}

\section{RESULTS AND DISCUSSION}

Results in Table (2) show that significant increase was detected in grain yield, plant height, No. of panicles/hill, No. of tillers/hill, panicle length, panicle weight and 1000 grain weight between $\mathrm{L}_{1}$ and $\mathrm{L}_{2}$ treatments. Laser land leveling $\left(\mathrm{L}_{2}\right)$ significantly increased grain yield, number of tillers/hill, number of panicles/hill, panicle length, plant height, Panicle weight and 1000 grains weight by $10.31 \%, 9.38 \%, 14.21 \%, 10.07 \%$, $7.84 \%, 4.43 \%$ and $5.42 \%$, respectively, compared with normal land leveling $\left(\mathrm{L}_{1}\right)$.

Planting in bottom of beds $\left(\mathrm{M}_{3}\right)$, significantly increased grain yield by $8.48 \%$, number of tillers/hill by $10.58 \%$, number of panicles/hill by $8.34 \%$, panicle length by $16.9 \%$, plant height by $4.38 \%$, panicle weight by $6.62 \%$ and 1000 grains weight by $6.83 \%$, compared with traditional planting method ( flat land) $\left(\mathrm{M}_{1}\right)$. These results coincided with those obtained by Khattak, et al. (2006), Mishra and Saha (2007), Jagroop et al. (2007) and El-Atawy (2012) who mentioned that grain yield of rice transplanted in bed produced high grain yield. 
Table (2): Average values of grain yield, plant height, number of panicles/hill, number of tillers/hill, panicle length, panicle weight and 1000-grain weight as influenced by land leveling, planting methods and irrigation depth.

\begin{tabular}{|c|c|c|c|c|c|c|c|c|c|}
\hline $\begin{array}{l}\text { Land } \\
\text { leveling }\end{array}$ & $\begin{array}{l}\text { Plant } \\
\text { method }\end{array}$ & $\begin{array}{l}\text { Irrig. } \\
\text { Depth } \\
\text { (cm) }\end{array}$ & $\begin{array}{c}\text { Grain } \\
\text { yield } \\
\text { (kg/ha.) }\end{array}$ & $\begin{array}{c}\text { Plant } \\
\text { height, } \\
\text { (cm) }\end{array}$ & $\begin{array}{c}\text { No. of } \\
\text { panicles/ } \\
\text { hill }\end{array}$ & $\begin{array}{c}\text { No. of } \\
\text { tillers/ } \\
\text { hill }\end{array}$ & $\begin{array}{l}\text { Panicle } \\
\text { length, } \\
\text { (cm) }\end{array}$ & $\begin{array}{l}\text { Panicle } \\
\text { Weight } \\
\text { (g) }\end{array}$ & $\begin{array}{c}1000 \\
\text { grain } \\
\text { Weight } \\
\text { (g) }\end{array}$ \\
\hline \multirow{9}{*}{$\left(\mathrm{L}_{1}\right)$} & \multirow{3}{*}{$\begin{array}{l}\text { Tradi- } \\
\text { tional } \\
\left(\mathbf{M}_{1}\right)\end{array}$} & $9\left(d_{1}\right)$ & $11.05 \mathrm{a}$ & 107 a & $25 \mathrm{a}$ & $27 \mathbf{a}$ & $21.5 \mathrm{a}$ & $2.70 \mathrm{a}$ & 27.89 a \\
\hline & & $7\left(d_{2}\right)$ & $11.00 \mathrm{a}$ & $105 \mathrm{~b}$ & 25 a & 26 a & 22 a & $2.62 \mathrm{a}$ & 27.29 b \\
\hline & & $5\left(d_{3}\right)$ & $7.82 \mathrm{~b}$ & $104 \mathrm{~b}$ & $20 \mathrm{~b}$ & $22 \mathrm{~b}$ & $18.5 \mathrm{~b}$ & $2.23 \mathrm{~b}$ & $24.14 \mathrm{c}$ \\
\hline & \multirow{3}{*}{$\begin{array}{l}\text { Furrow } \\
\left(\mathbf{M}_{2}\right)\end{array}$} & $9\left(d_{1}\right)$ & $11.71 \mathrm{a}$ & 110 a & 26 a & 28 a & $22.5 \mathrm{ab}$ & $2.79 \mathrm{a}$ & 28.04 a \\
\hline & & $7\left(d_{2}\right)$ & $11.58 \mathrm{a}$ & 112 a & $27 \mathrm{a}$ & $28 \mathrm{a}$ & 23.5 a & $2.75 \mathrm{a}$ & 28.40 a \\
\hline & & $5\left(d_{3}\right)$ & $8.18 \mathrm{~b}$ & $107 \mathrm{~b}$ & $21 \mathrm{~b}$ & $24 \mathrm{~b}$ & 20.51 b & $2.31 \mathrm{~b}$ & $25.74 a$ \\
\hline & \multirow{3}{*}{$\begin{array}{l}\text { Bed } \\
\left(\mathbf{M}_{3}\right)\end{array}$} & $9\left(d_{1}\right)$ & 11.85 a & 112 a & $27 \mathrm{a}$ & 29 a & $24 \mathrm{ab}$ & 2.87 a & 28.81 a \\
\hline & & $7\left(d_{2}\right)$ & 11.89 a & $114 \mathrm{a}$ & 28 a & $30 \mathrm{a}$ & 25 a & $2.79 \mathrm{a}$ & 29.45 a \\
\hline & & $5\left(d_{3}\right)$ & $8.30 \mathrm{~b}$ & $109 \mathrm{~b}$ & $22 \mathrm{~b}$ & $25 \mathrm{~b}$ & $21.5 \mathrm{~b}$ & $2.35 \mathrm{~b}$ & 26.02 b \\
\hline \multicolumn{2}{|c|}{ Mean $\left(\mathrm{L}_{1}\right)$} & & 10.37 & 108.9 & 24.6 & 26.6 & 22.1 & 2.60 & 27.25 \\
\hline \multirow{9}{*}{$\left(\mathbf{L}_{2}\right)$} & \multirow{3}{*}{$\begin{array}{c}\text { Tradi- } \\
\text { tional } \\
\left(\mathbf{M}_{1}\right)\end{array}$} & $9\left(d_{1}\right)$ & 11.31a & 109 a & 28 a & 29 a & $22.5 \mathrm{~b}$ & $2.81 \mathrm{a}$ & $28.10 \mathrm{~b}$ \\
\hline & & $7\left(d_{2}\right)$ & $11.23 \mathrm{a}$ & $110 \mathrm{a}$ & 28 a & 28 a & $23.5 \mathrm{a}$ & $2.77 \mathrm{a}$ & 28.50 a \\
\hline & & $5\left(d_{3}\right)$ & $9.55 \mathrm{~b}$ & $105 \mathrm{~b}$ & $25 \mathrm{~b}$ & $26 \mathrm{~b}$ & $19.5 \mathrm{c}$ & $2.31 \mathrm{~b}$ & $26.66 \mathrm{c}$ \\
\hline & \multirow{3}{*}{$\begin{array}{l}\text { Furrow } \\
\left(\mathbf{M}_{2}\right)\end{array}$} & $9\left(d_{1}\right)$ & $11.91 \mathrm{a}$ & $111 \mathrm{a}$ & 29 a & 30 a & $26.5 \mathrm{a}$ & $2.88 \mathrm{a}$ & 29.05 a \\
\hline & & $7\left(d_{2}\right)$ & $12.33 \mathrm{a}$ & 112 a & $30 \mathbf{a}$ & 30 a & 26 a & $2.86 \mathrm{a}$ & 29.22 a \\
\hline & & $5\left(d_{3}\right)$ & 10.47 a & $107 \mathrm{~b}$ & $26 \mathrm{~b}$ & $27 \mathrm{~b}$ & $22.5 \mathrm{~b}$ & $2.39 \mathrm{~b}$ & 27.09 a \\
\hline & \multirow{3}{*}{$\begin{array}{l}\text { Bed } \\
\left(\mathbf{M}_{3}\right)\end{array}$} & $9\left(d_{1}\right)$ & 11.97a & $112 \mathrm{a}$ & $30 \mathbf{a}$ & 31 a & $27 \mathbf{a}$ & $2.95 \mathrm{a}$ & 30.78 a \\
\hline & & $7\left(d_{2}\right)$ & $12.51 \mathrm{a}$ & 113 a & 31 a & 32 a & 28 a & $2.98 \mathrm{a}$ & 30.91 a \\
\hline & & $5\left(d_{3}\right)$ & 10.67 a & $108 \mathrm{~b}$ & $27 \mathrm{~b}$ & $28 \mathrm{~b}$ & $23.5 \mathrm{~b}$ & $2.51 \mathrm{~b}$ & $28.23 \mathrm{a}$ \\
\hline \multicolumn{3}{|c|}{ Mean $\left(\mathrm{L}_{2}\right)$} & 11.33 & 109.70 & 28.2 & 29.0 & 24.34 & 2.72 & 28.73 \\
\hline \multicolumn{3}{|c|}{ LSD at 0.05} & 1.3 & 0.016 & 0.9 & 0.9 & 1.0 & 0.0095 & $\begin{array}{c}0.049 \\
\end{array}$ \\
\hline \multirow{3}{*}{\multicolumn{2}{|c|}{$\begin{array}{l}\text { Means values of } \\
\text { Planting methods }\end{array}$}} & 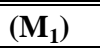 & $10.32 \mathrm{~b}$ & $106.67 \mathrm{~b}$ & $25.17 b$ & $26.3 b$ & $21.25 \mathrm{~b}$ & 27.10 b & $27.10 \mathrm{~b}$ \\
\hline & & $\left(M_{2}\right)$ & $11.03 \mathrm{a}$ & $109.83 a$ & 26.50a & $27.8 \mathrm{a}$ & 23.85 a & 27.92 a & 27.92 a \\
\hline & & $\left.\mathbf{M}_{3}\right)$ & $11.20 \mathrm{a}$ & 111.33a & 27.50a & $29.2 \mathrm{a}$ & $24.84 \mathrm{a}$ & 28.95 a & $28.95 \mathrm{a}$ \\
\hline \multicolumn{3}{|c|}{ LSD at 0.05} & 1.2 & $\begin{array}{c}0.015 \\
\end{array}$ & 0.6 & 0.7 & 0.9 & $\begin{array}{l}0.0084 \\
\end{array}$ & $\begin{array}{l}0.053 \\
\end{array}$ \\
\hline \multirow{3}{*}{\multicolumn{2}{|c|}{$\begin{array}{l}\text { Means values of } \\
\text { irrigation depth }\end{array}$}} & $9\left(d_{1}\right)$ & $111.65 a$ & $\begin{array}{l}110.17 a \\
\end{array}$ & 27.50a & $29.0 a$ & 24.00 a & 28.78 a & 28.78 a \\
\hline & & $7\left(d_{2}\right)$ & $11.76 a$ & 111.00a & $28.17 a$ & $29.0 a$ & $24.67 a$ & 28.88 a & 28.88 a \\
\hline & & $5\left(d_{3}\right)$ & $9.17 b$ & $106.67 \mathrm{~b}$ & 23.50b & $25.3 \mathrm{~b}$ & $21.50 \mathrm{~b}$ & $26.32 \mathrm{~b}$ & $26.32 \mathrm{~b}$ \\
\hline \multicolumn{3}{|c|}{ LSD at 0.05} & 1.3 & 0.015 & 0.7 & 1.2 & 0.8 & 0.0095 & 0.050 \\
\hline \multicolumn{3}{|c|}{$\bar{L} \mathrm{x}$ season } & ns & ns & ns & ns & ns & ns & ns \\
\hline \multicolumn{3}{|c|}{ M x season } & ns & ns & ns & ns & ns & ns & ns \\
\hline \multicolumn{3}{|c|}{ d $x$ season } & ns & ns & ns & ns & ns & ns & ns \\
\hline \multicolumn{3}{|l|}{ L X M } & $* *$ & $* *$ & $* *$ & $* *$ & $* *$ & $* *$ & $* *$ \\
\hline \multicolumn{3}{|l|}{ Lxd } & $* *$ & $* *$ & $* *$ & $* *$ & $* *$ & $* *$ & $* *$ \\
\hline \multicolumn{3}{|l|}{ Mxd } & $* *$ & $* *$ & $* *$ & $* *$ & $* *$ & $* *$ & $* *$ \\
\hline \multicolumn{3}{|c|}{$L \times M \times d$} & $* *$ & $* *$ & $* *$ & $* *$ & $* *$ & $* *$ & $* *$ \\
\hline
\end{tabular}

No significant differences in all studied characters between irrigation 
depth $9 \mathrm{~cm}\left(\mathrm{~d}_{1}\right)$ treatment and irrigation depth $7 \mathrm{~cm}\left(\mathrm{~d}_{2}\right)$. As for the effect of the deficit irrigation treatments on the studied characters, the obtained results showed that irrigation depth $7 \mathrm{~cm}\left(\mathrm{~d}_{2}\right)$ significantly increased grain yield by $28.32 \%$, number of tillers/hill by $13.69 \%$, number of panicles/hill by $19.28 \%$, panicle length by $17.5 \%$, plant height by $3.83 \%$, panicle weight by $18.94 \%$ and 1000 grains weight by $9.73 \%$, compared to irrigation depth $5 \mathrm{~cm}\left(\mathrm{~d}_{3}\right)$. The higher grain yield of $\mathrm{d}_{1}$ and $\mathrm{d}_{2}$ treatments than that of $d_{3}$ could be attributed to the high yield components such as the number of grains per panicle, panicle weight, and panicle length of treatment $d_{1}$ and $d_{2}$, as shown in Table (2). These results coincided with those obtained by Atta et al. (2006), Khattak, et al. (2006), Mishra and Saha (2007), Jagroop et al. (2007) and El-Atawy (2012).

Insignificant effect of planting method and season interaction was obtained from all traits. Such results indicated that irrigation depth treatments showed similar effect from season to season. Data in Table (2) show that the average values of grain yield, plant height, No. of panicles/hill, No. of tillers/hill, panicle length, panicle weight and 1000 grain weight were high significantly affected by the interaction between land leveling treatments(L), planting methods (M) and irrigation depth (d). It is obvious from Table (2) that the highest mean values of grain yield, plant height, No. of panicles/hill, No. of tillers/hill, panicle length, panicle weight and 1000 grain weight were obtained from $L_{2} \times M_{3} \times d_{2}$, whereas, the lowest value of all studied characters were obtained from $L_{1}$ $x M_{1} \times d_{3}$. These results could be attributed to the interaction effect between precision land leveling, irrigation depth and transplanting methods. Impact of irrigation depth on yield and its components under different planting methods was in descending order $M_{3}>M_{2}>M_{1}$. This indicates that irrigation depth was more influential on $\mathrm{M}_{3}$ (bed) than on the other planting methods. These results are in agreement with those obtained by Atta et al. (2006), Mishra and Saha (2007) and El-Atawy (2012).

\section{Irrigation water applied (IWA)}

Seasonal water applied in $\mathrm{m}^{3} \mathrm{ha}^{-1}$ as affected by land leveling, planting methods and irrigation depth, is presented in Table 3. There are significant differences in total amounts of water applied between 
irrigation depths 9,7 and $5 \mathrm{~cm}\left(\mathrm{~d}_{1}, \mathrm{~d}_{2}\right.$ and $\left.\mathrm{d}_{3}\right)$ in all planting method $\left(\mathrm{M}_{1}\right.$, $\mathrm{M}_{2}$ and $\left.\mathrm{M}_{3}\right)$ and both land leveling $\left(\mathrm{L}_{1}\right.$ and $\left.\mathrm{L}_{2}\right)$.

Table (3): Grain yield $\left(\mathrm{Kg} \mathrm{ha}^{-1}\right)$, seasonal water applied (WA in $\mathrm{m}^{3} \mathrm{ha}^{-1}$ ) and productivity of irrigation water (PIW) $\mathrm{Kg} \mathrm{m}^{-3}$ ) as affected by land leveling, planting methods and irrigation depth in combined analysis of 2012 and 2013 seasons.

\begin{tabular}{|c|c|c|c|c|c|}
\hline $\begin{array}{l}\text { Land } \\
\text { leveling }\end{array}$ & Plant method & $\begin{array}{l}\text { Irrig. } \\
\text { depth }\end{array}$ & $\begin{array}{l}\text { Grain yield } \\
\text { kg/ha. }\end{array}$ & $\begin{array}{c}\text { Water } \\
\text { applied }\end{array}$ & $\underset{\left(\mathrm{Kg} \mathrm{m}^{-3}\right)}{\text { PIW }}$ \\
\hline \multirow{9}{*}{$\left(\mathrm{L}_{1}\right)$} & \multirow{3}{*}{$\begin{array}{l}\text { Tradi- } \\
\text { tional } \\
\left(M_{1}\right)\end{array}$} & $9\left(d_{1}\right)$ & 11.09 a & 17008 a & $0.652 \mathrm{c}$ \\
\hline & & $7\left(d_{2}\right)$ & $11.03 \mathrm{a}$ & $15093 \mathrm{~b}$ & 0.731 a \\
\hline & & $5\left(d_{3}\right)$ & $7.84 \mathrm{~b}$ & $11413 \mathrm{c}$ & 0.687 b \\
\hline & \multirow{3}{*}{$\begin{array}{l}\text { Furrow } \\
\left(\mathbf{M}_{2}\right)\end{array}$} & $9\left(d_{1}\right)$ & $11.73 \mathrm{a}$ & 13825 a & 0.848 a \\
\hline & & $7\left(d_{2}\right)$ & $11.60 \mathrm{a}$ & $12205 \mathrm{~b}$ & 0.950 a \\
\hline & & $5\left(d_{3}\right)$ & $8.19 \mathrm{~b}$ & $9822 \mathrm{c}$ & $0.834 \mathrm{a}$ \\
\hline & \multirow{3}{*}{$\begin{array}{l}\text { Bed } \\
\left(\mathbf{M}_{3}\right)\end{array}$} & $9\left(d_{1}\right)$ & $11.86 \mathrm{a}$ & 10439 a & $1.136 \mathrm{a}$ \\
\hline & & $7\left(d_{2}\right)$ & $11.92 \mathrm{a}$ & $9202 \mathrm{~b}$ & $1.295 \mathrm{a}$ \\
\hline & & $5\left(d_{3}\right)$ & $8.30 \mathrm{~b}$ & $7389 \mathrm{c}$ & $1.123 \mathrm{a}$ \\
\hline \multicolumn{3}{|c|}{ Mean $\left(\mathrm{L}_{1}\right)$} & 10.40 & 11822 & 0.880 \\
\hline \multirow{9}{*}{$\left(\mathbf{L}_{2}\right)$} & \multirow{3}{*}{$\begin{array}{l}\text { Tradi- } \\
\text { tional } \\
\left(M_{1}\right)\end{array}$} & $9\left(d_{1}\right)$ & $11.32 \mathrm{a}$ & 15483 a & $0.731 \mathrm{c}$ \\
\hline & & $7\left(d_{2}\right)$ & $11.24 \mathrm{a}$ & $13626 \mathrm{~b}$ & $0.825 \mathrm{~b}$ \\
\hline & & $5\left(d_{3}\right)$ & $9.56 \mathrm{~b}$ & $10689 \mathrm{c}$ & $0.894 \mathrm{a}$ \\
\hline & \multirow{3}{*}{$\begin{array}{l}\text { Furrow } \\
\left(\mathbf{M}_{2}\right)\end{array}$} & $9\left(d_{1}\right)$ & $11.92 \mathrm{a}$ & 13049 a & $0.913 \mathrm{a}$ \\
\hline & & $7\left(d_{2}\right)$ & $12.34 \mathrm{a}$ & $11631 \mathrm{~b}$ & $1.061 \mathrm{a}$ \\
\hline & & $5\left(d_{3}\right)$ & $10.48 \mathrm{a}$ & $9182 \mathrm{c}$ & $1.141 \mathrm{a}$ \\
\hline & \multirow{3}{*}{$\begin{array}{l}\text { Bed } \\
\left(\mathbf{M}_{3}\right)\end{array}$} & $9\left(d_{1}\right)$ & $11.98 \mathrm{a}$ & 10174 a & $1.178 \mathrm{a}$ \\
\hline & & $7\left(d_{2}\right)$ & $12.52 \mathrm{a}$ & $9104 \mathrm{~b}$ & $1.375 \mathrm{a}$ \\
\hline & & $5\left(d_{3}\right)$ & 10.69 a & $7209 \mathrm{c}$ & 1.483 \\
\hline \multicolumn{3}{|c|}{ Mean $\left(\mathbf{L}_{2}\right)$} & 11.34 & 11127 & 1.019 \\
\hline \multicolumn{3}{|c|}{ LSD 0.05 } & 1.1 .3 & 79.5 & 0.0058 \\
\hline \multirow{3}{*}{\multicolumn{2}{|c|}{$\begin{array}{l}\text { Means values of } \\
\text { Planting methods }\end{array}$}} & 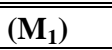 & $10.32 \mathrm{~b}$ & 13885 a & $0.743 \mathrm{c}$ \\
\hline & & $\left(M_{2}\right)$ & $11.03 \mathrm{a}$ & $11619 \mathrm{~b}$ & 0.949 b \\
\hline & & $\left.\mathbf{M}_{3}\right)$ & $11.20 \mathrm{a}$ & 8919 c & $1.256 \mathrm{a}$ \\
\hline \multicolumn{3}{|c|}{ LSD 0.05} & 1.2 & 86.4 & 0.0042 \\
\hline \multirow{3}{*}{\multicolumn{2}{|c|}{$\begin{array}{l}\text { Means values of } \\
\text { irrigation depth }\end{array}$}} & $9\left(d_{1}\right)$ & $11.65 \mathrm{a}$ & 13330 a & 0.874 b \\
\hline & & $7\left(d_{2}\right)$ & $11.76 \mathrm{a}$ & $11810 \mathrm{~b}$ & 0.996 a \\
\hline & & $5\left(d_{3}\right)$ & $9.17 \mathrm{~b}$ & $9284 \mathrm{c}$ & 0.988 a \\
\hline \multicolumn{3}{|c|}{ LSD 0.05 } & 1.3 & 81.6 & 0.0048 \\
\hline \multicolumn{3}{|c|}{ L x season } & ns & Ns & ns \\
\hline \multicolumn{3}{|c|}{ M x season } & ns & Ns & ns \\
\hline \multicolumn{3}{|c|}{ d $x$ season } & ns & Ns & ns \\
\hline \multicolumn{3}{|c|}{$L \times M$} & $* *$ & $* *$ & $* *$ \\
\hline \multicolumn{3}{|l|}{ L x d } & $* *$ & $* *$ & $* *$ \\
\hline \multicolumn{3}{|l|}{ Mxd } & $* *$ & $* *$ & $* *$ \\
\hline \multicolumn{3}{|c|}{$L \times M \times d$} & $* *$ & $* *$ & $* *$ \\
\hline
\end{tabular}


It is clear that the highest total amount of water applied are $17008 \mathrm{~m}^{3}$ ha1 , resulted from irrigation depth $9 \mathrm{~cm}\left(\mathrm{~d}_{1}\right)$ under traditional planting method $\left(\mathrm{M}_{1}\right)$ and normal land leveling $\left(\mathrm{L}_{1}\right)$, while the lowest total amount of water applied are $7209 \mathrm{~m}^{3}$ ha- ${ }^{1}$, result from irrigation depth $5 \mathrm{~cm}\left(\mathrm{~d}_{3}\right)$ in beds planting method $\left(\mathrm{M}_{3}\right)$ and laser land leveling $\left(\mathrm{L}_{2}\right)$. These results declare that the laser land leveling saved irrigation water by $57.6 \%$, compared to normal land leveling. On the other hand the beds planting method $\left(\mathrm{M}_{3}\right)$ saves irrigation water by $35.8 \%$ compared to $\left(\mathrm{M}_{1}\right)$, while, irrigation depth $5 \mathrm{~cm}\left(\mathrm{~d}_{3}\right)$ saves irrigation water by $30.4 \%$, compared to irrigation depth $9 \mathrm{~cm}\left(\mathrm{~d}_{1}\right)$. Generally, the treatment $\mathrm{L}_{2} \times \mathrm{M}_{3} \times \mathrm{d}_{3}$ saved irrigation water by $57.6 \%$, compared to treatment $\mathrm{L}_{1} \times \mathrm{M}_{1} \times \mathrm{d}_{1}$, but it decreases grain yield by $3.6 \%$.

It is obvious that the amount of irrigation water applied was gradually increased as a result of the growing up to vegetative growth that required high amount of irrigation water to meet its water requirements, and then it decreased again. These findings may be attributed to growth stage and weather conditions accompanying growth stage. These results are in agreement with those obtained by Atta et al. (2006), Mishra and Saha (2007), Meleha et al. (2008) and El-Atawy (2012).

\section{Productivity of irrigation water (PIW)}

Mean values of PIW of rice ( $\mathrm{kg}$ grain $\mathrm{m}^{-3}$ ) as affected by land leveling, planting methods and irrigation depth in combined analysis of 2012 and 2013 seasons, are presented in Table 3. Results showed that no significant differences in productivity of irrigation water (PIW) between irrigation depths $9 \mathrm{~cm}\left(\mathrm{~d}_{1}\right), 7 \mathrm{~cm}\left(\mathrm{~d}_{2}\right)$ and $5 \mathrm{~cm}\left(\mathrm{~d}_{3}\right)$ under transplanting in furrow $\left(\mathrm{M}_{2}\right)$ and transplanting in beds $\left(\mathrm{M}_{3}\right)$, while with traditional planting method $\left(\mathrm{M}_{1}\right)$ there are significant differences in (PIW) values between irrigation depths. Results showed that $\mathrm{M}_{3}$ treatment increased PIW by $13 \%$ more than $\mathrm{M}_{1}$ treatments. Similar results were reported by Vethaiya et al. (2003), Atta (2005), Atta et al. (2006), Choudhury et al. (2007), and El-Atawy (2012).

The interaction between treatments of land leveling (L), planting methods (M) and irrigation depth (d) (Table 3) shows that the highest PIW are $1.483 \mathrm{~kg}$ grain $\mathrm{m}^{-3}$, resulted from irrigation depth $5 \mathrm{~cm}\left(\mathrm{~d}_{3}\right)$ under beds 
planting method $\left(\mathrm{M}_{3}\right)$ and laser land leveling $\left(\mathrm{L}_{2}\right)$. while the lowest values of PIW were $0.652 \mathrm{~kg}$ grain $\mathrm{m}^{-}$, resulted from irrigation depth $9 \mathrm{~cm}\left(\mathrm{~d}_{1}\right)$ under traditional planting method $\left(\mathrm{M}_{1}\right)$ and normal land leveling $\left(\mathrm{L}_{1}\right)$. These results could be attributed to the significant differences among grain yield, and to the irrigation water applied values. Values of grain yield of $d_{1}$ treatment was much higher than that of $d_{2}$ and $d_{3}$ treatments and the irrigation water applied of $\mathrm{d}_{2}$ and $\mathrm{d}_{3}$ treatments were less than that of $d_{1}$ treatment (see Table 3). Similar results were reported by Vethaiya $\boldsymbol{e t}$ al. (2003), Atta (2005), Atta et al. (2006), Choudhury et al. (2007), and El-Atawy (2012).

\section{CONCLUSION}

It is necessary to produce more rice with less water by using new planting methods and less submerged head of irrigation water. The obtained results of the current study indicate that irrigation water applied in rice fields could be significantly reduced without sacrificing rice yield or without increasing the production cost by using the treatment $\mathrm{d}_{3} \times \mathrm{M}_{3} \times \mathrm{L}_{2}$. Method of transplanting at bottom of beds $\left(\mathrm{M}_{3}\right)$ increased PIW by $13 \%$ than $\mathrm{M}_{1}$. Therefore, transplanting rice in beds under laser land leveling and keeping it under continuous irrigation $\left(\mathrm{d}_{1} \times \mathrm{M}_{2} \times \mathrm{L}_{2}\right)$ could be applied by the farmers because it increased PIW by $13 \%$ and saved irrigation water by $35.8 \%$ compared to $\mathrm{d}_{1} \times \mathrm{M}_{1} \times \mathrm{L}_{2}$ in North Delta, Egypt. Transplanting rice in beds $\left(\mathrm{M}_{3}\right)$ and laser leveling $\left(\mathrm{L}_{2}\right)$ only was better than the other methods because there is no significant difference between $M_{1}$ and $M_{3}$ in grain yield and gave the highest PIW.

\section{REFERENCES}

Ali, M. H.; M. R. Hoque; A. A. Hassa and A. Khair (2007). Effect of deficit irrigation on yield, water productivity and economic returns of wheat. Agric.Water Manag. 92:151-161.

Atta, Y. I. M. (2005). Strip planting of rice: A new method for increasing water use efficiency under splitting of nitrogen fertilizer. Egypt. J. of Appl. Sci. 20 (10B): 501-511.

Atta, Y. I. M.; M. E.Meleha; A. Tallet and U. M.Gawish (2006). Improving water productivity in rice cultivation with high potential for water saving. The $3^{\text {rd }}$ Arab world region conference, Cairo. 
Beecher, H. G.; B. W. Dunn ; J. A. Thompson ; E. Humphreys ; S. K. Mathews and J. Timsina (2006). Effect of raised beds, irrigation and nitrogen management on growth, water use and yield of rice in south-eastern Australia. Australian J. of Experimental Agriculture. 46 (10): 1363-1372.

Choudhury B. U.; B. A. M. Bouman and A. K.Singh (2007). Yield and water productivity of rice-wheat on raised beds, results from a field experiment at New Delhi, India Field Crops Res. 100, 229-239.

El-Atawy, Gh. Sh. (2012). Saving Irrigation Water and Improving Water Productivity in Rice Cultivation by Inducing New Planting Method in North Delta, Egypt. J. Soil Sci. and Agric. Engineering, Mansoura Univ., 3 (5): 587-599.

Gomez, K. A. and A. A. Gomez (1984). Statistical procedures for agricultural research. Johns Willey and Sons. Inc. New York, USA.

Jagroop K.; Mahey R. K.; Vashist K.K. and Mahal S.S. (2007). Growth and productivity of rice (Oryza sativa L.) and water expense efficiency as influenced by different planting techniques. Environment and Ecology. 25(1): 235-238.

Khattak S. I.; K. Usman, Q. Khan and A. Qayyum (2006). Impact of various planting techniques on yield and yield components of rice. Indus J. of Plant Sciences. 5(1): 753-756.

Meleha, M. E.; A.Z. El-Bably; A. A. Abd Allah and W. M. El-Khoby (2008). Producing more rice with less water by inducing planting methods in north Delta, Egypt. J. Agric. Sci., Mansoura Univ., 33 (1): 805-813.

Mishra, V. and R. Saha (2007). Effect of raised sunken bed system on inter-plot water harvesting and productivity of rice and French bean in Meghalaya. Indian J. of Agric. Sci. 77 (2): 73-78.

Molden, D.; R.Sakthivadivel and Z. Habib (2001). Basin-level use and productivity of water: examples from South Asia. Research report 49, International Water Management Institute (IWMI), Colombo, Sri Lanka, : 24.

Rijsberman, F. (2001). Can the CGIAR solve the world water crisis? 
Paper presented at the CGIAR mid-term meeting 2001 in Durban, South Africa, : 7.

Vethaiya B.; J. K. Ladha; K. R. Gupta; R. K. Naresh; R. S. Mehla and S. Yadvinder (2003). Technology options for rice in the rice wheat rotation in South Asia. Improving the productivity and sustainability of rice wheat rotation: Issues and Impacts Proceedings of an International Sym., USA, 22 October, 115-147.

Vories, E. D.; P. A. Counce; and T. C. Keisling. (2002). Comparison of flooded and furrow-irrigated rice on clay. Irrig. Sci. 21:139-144.

Waller, R. A. and D. B. Duncan. (1969). Symmetric multiple Comparison Problem. Amer. Stat.Assoc. Jour.December, 1485-1503.

\section{الملخص العربي}

توفير مياه الري وتحسين إنتاجيتها لمحصول الأرز باستخدام التسوية الجيدة للتربة وطرق زراعة جديدة في شمال دلتا التهائ التيل

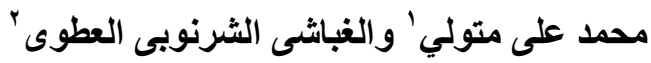

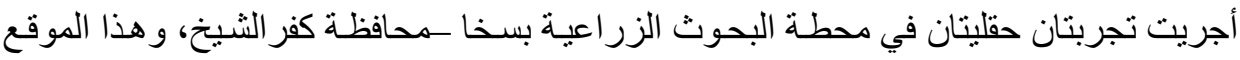

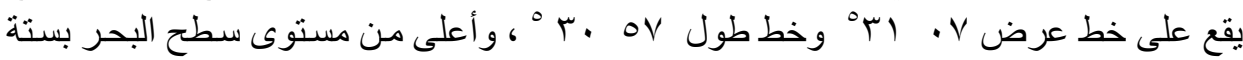

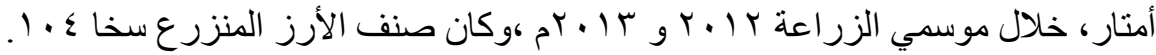

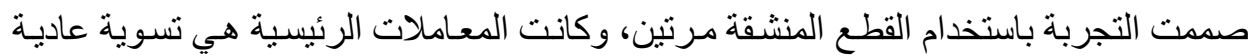

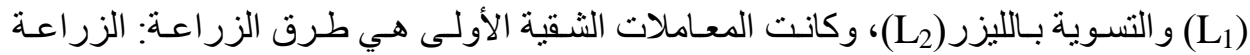

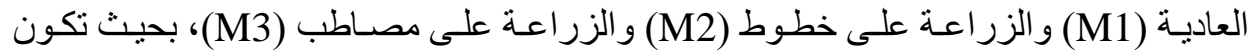

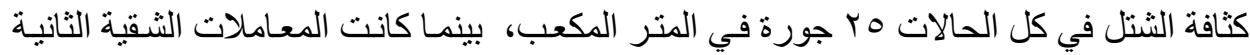

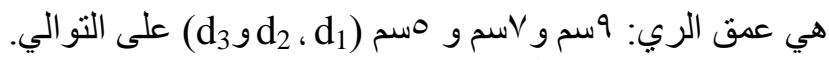

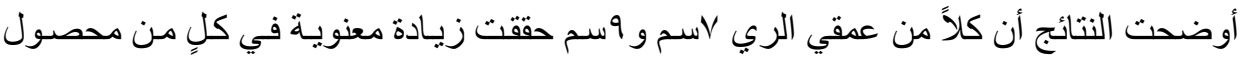

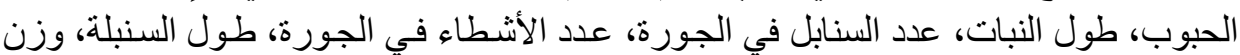

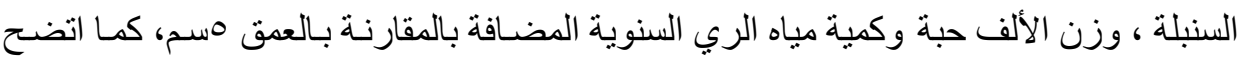

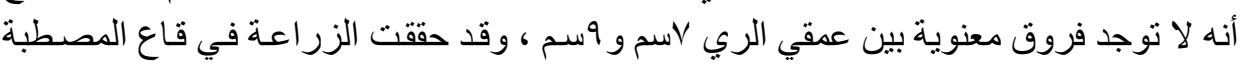

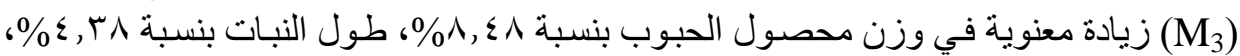

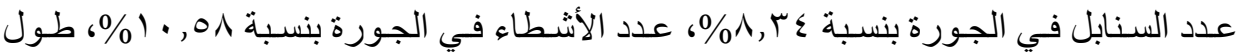

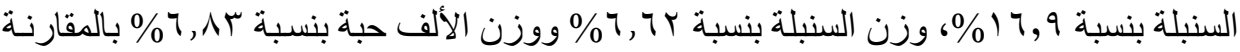
بالزر اعة العادية (M) (M)

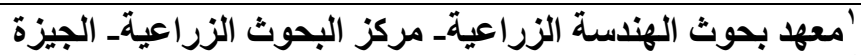

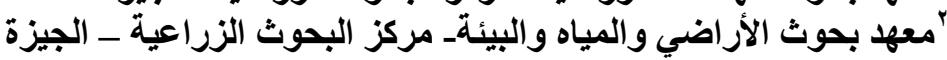




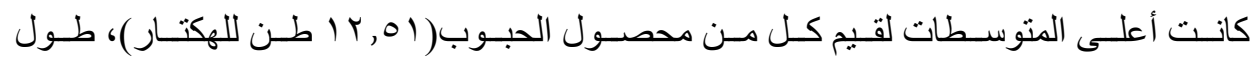

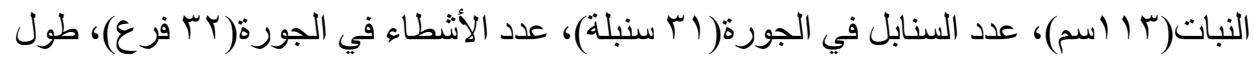

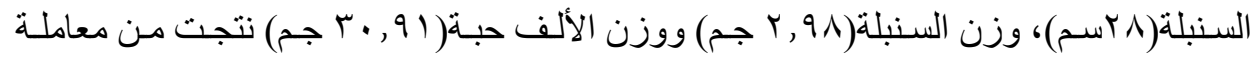
التسوية بالليزر مع الزر اعة على مصاطب و الري بعمق لسم (d (d )

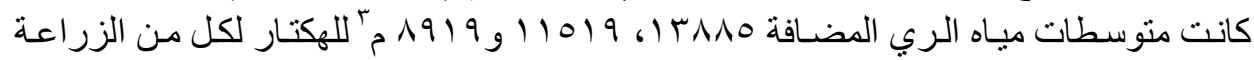

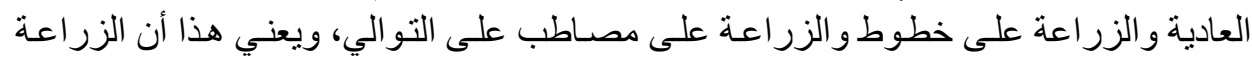

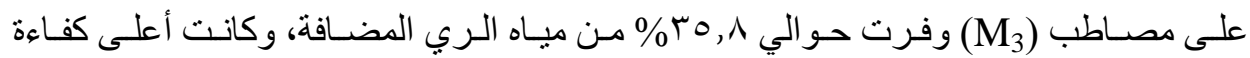

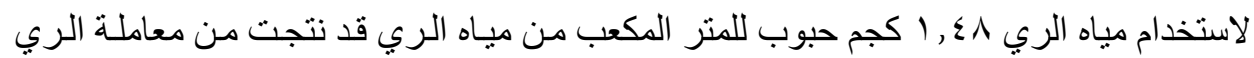

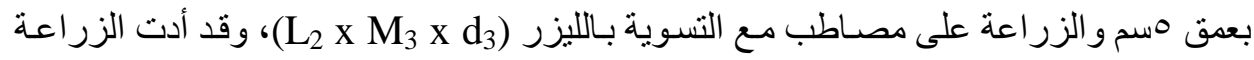

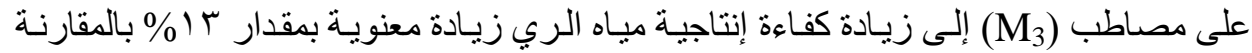

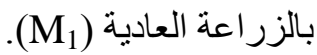
لهذا بمكن التوصية بزراعة شتادلات الأرز على مصاطب في شمال دلتا النيل لأنها ترفع كفاءة

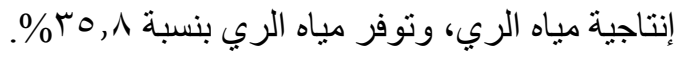

